\title{
NeuroRegulation
}

\section{Which Quiets the Mind More Quickly and Increases HRV: Toning or Mindfulness?}

\author{
Erik Peper1, Weston Pollock ${ }^{1}$, Richard Harvey ${ }^{1}$, Aiko Yoshino ${ }^{1}$, Jennifer Daubenmier ${ }^{1}$, and \\ Madhu Anziani²
}

\author{
${ }^{1}$ Institute for Holistic Health Studies, San Francisco State University, San Francisco, California, USA \\ ${ }^{2}$ Firstwasthesound, Richmond, California, USA
}

\begin{abstract}
Disruptive thoughts interfere with concentration and performances. This report compares mindfulness practice (MP) with toning practice (TP) to reduce mind wandering and intrusive thoughts. Ninety-one undergraduate students (average age 22.4 years) began with either an MP or TP for $3 \mathrm{~min}$. Respiration, blood volume pulse, and heart rate were monitored for 11 participants. The participants reported less mind wandering during TP $(M=$ 3.7) than during MP $(M=6.5 ; p<.001)$, fewer intrusive thoughts during TP $(M=3.2)$ than during MP $(M=4.7 ; p$ $<.001)$, and more body vibrations during TP $(M=6.2)$ than during MP $(M=2.3 ; p<.001$ ) on a scale from 0 (not at all) to 10 (all the time). For participants with the highest self-reported rating of depression, TP was more effective in reducing mind wandering and intrusive thoughts than the MP $(p<.001)$. There was no difference in self-reports in peacefulness, warmth, relaxation, anxiety, and depression between TP and MP. There was a decrease in respiration rate during TP $(4.6 \mathrm{br} / \mathrm{min})$ as compared to MP $(11.6 \mathrm{br} / \mathrm{min} ; p<.001)$ and an increase in heart rate variability during TP $(S D N N=103.7 \mathrm{~ms} ; S D=11.6)$ as compared to MP (SDNN $=61.9 \mathrm{~ms} ; S D=6.4)$. The findings suggest that TP is a powerful strategy to reduce mind wandering and intrusive thoughts.

Keywords: toning; mindfulness meditation; respiration; heart rate variability; intrusive thoughts; wandering thoughts; peacefulness; relaxation; depression

Citation: Peper, E., Pollock, W., Harvey, R., Yoshino, A., Daubenmier, J., \& Anziani, M. (2019). Which quiets the mind more quickly and increases HRV: Toning or mindfulness? NeuroRegulation, 6(3), 128-133. https://doi.org/10.15540/nr.6.3.128

*Address correspondence to: Erik Peper, PhD, Institute for Holistic Healing Studies, Department of Health Education, San Francisco State University, 1600 Holloway Avenue, San Francisco, CA 94132, USA. Email: epeper@sfsu.edu

Copyright: (c) 2019. Peper et al. This is an Open Access article distributed under the terms of the Creative Commons Attribution License (CC-BY).

Edited by:

Rex L. Cannon, PhD, SPESA Research Institute, Knoxville, Tennessee, USA

Reviewed by:

Randall Lyle, PhD, Mount Mercy University, Cedar Rapids, lowa, USA

Nancy L. Wigton, PhD, Grand Canyon University, Phoenix, Arizona, USA
\end{abstract}

\section{Background}

Intrusive thoughts and worries are common experiences that are often challenging to control, and sometimes interfere with learning self-regulation and mastering bio-neurofeedback skills. Mindfulness practice (MP) and heart rate variability (HRV) training have been combined by some practitioners with neurofeedback training as an integrated approach for enhancing emotional and physiological self-regulation, and for achieving sympathetic and parasympathetic balance (Thompson \& Thompson, 2015). Numerous clinical strategies are taught to clients to reduce negative ruminations such as third-wave cognitive behavioral therapy (CBT) as described by Kopelman-Rubin,
Omer, and Dar (2017). In this context, third-wave CBT considers how people relate to their thoughts and emotions rather than the exact content of those thoughts and emotions, such as using emotion regulation therapy (Renna, Quintero, Fresco, \& Mennin, 2017) and more recently mindfulness meditation/training (Hofmann \& Gomez, 2017; Ost, 2008). Although participants often become successful in learning to relate better to their thoughts and emotions using these third-wave techniques, many continue to struggle with distracting or wandering thought processes. Letting go of worrying thoughts and rumination is even more challenging when one is upset, angry, or captured by stressful life circumstances. It may be possible that other strategies can more rapidly reduce 
wandering and intrusive thoughts. For example, van der Zwan, de Vente, Huizink, Bogels, and de Bruin (2015) found that physical activity (PA), mindfulness meditation (MM; in contrast to mindfulness-based $\mathrm{CBT}$ ), and heart rate variability biofeedback (HRVBF) for 5 weeks' practice are equally effective in reducing stress and its related symptoms when compared to typical psychotherapies. In addition, meditators often report that mantra meditation, singing, chanting, and toning can quickly quiet the mind.

Recently, MM has been integrated and adapted as one major strategy for reducing wandering thoughts during bio- and neurofeedback training (Khazan, 2013, 2019). Within the context of the techniques described by Khazan (2013), mindfulness training consists of instructions for attending to presentmoment experiences such as the experience of breathing, repeating a mantra, or imagining some visual object, as well as embracing an attitude of nonjudgmental acceptance and awareness of present-moment thoughts. As negative-emotion thoughts arise, the instruction is to recognize the thoughts without judging or becoming experientially "fused" with them in a process referred to as "metaawareness" (Dahl, Lutz, \& Davidson, 2015) before "disengaging" from those negative thoughts, and then turning attention back to the experiences of training and raising awareness of the intended goal of the training. Mindfulness training combined with bio- and neurofeedback training has been shown to improve a wide range of psychological and physical health conditions associated with symptoms of stress, such as anxiety, depression, chronic pain, and addiction (Creswell, 2015).

Biofeedback-assisted HRV training can be very effective to encourage sympathetic-parasympathetic balance (Lagos, Thompson, \& Vaschillo, 2013; Lehrer \& Gevirtz, 2014; McGrady \& Moss, 2018; Shaffer \& Ginsberg, 2017). It is a highly beneficial approach for the treatment of anxiety, gastrointestinal distress, posttraumatic stress, and concussions (Condor \& Condor, 2014; Ginsberg, Berry, \& Powell, 2010; Goessl, Curtiss, \& Hofmann, 2017; Lagos et al., 2013; Lehrer \& Gevirtz, 2014). The biofeedback-assisted training focuses on increasing HRV typically through breathing pacing at a rate of about 6 breaths per minute.

In a randomized control trial (RCT), van der Zwan et al. (2015) compared the efficacy of self-help PA, $\mathrm{MM}$, and HRV-BF training over 5 weeks of practice to reduce stress and its related symptoms. They found that PA, MM, and HRV-BF training were equally effective in reducing their measurements of stress and its related symptoms. Even though people can learn to practice PA, MM, and HRV-BF techniques, many continue to struggle, although less often, with distracting or wandering thought processes. Letting go of worrying thoughts and rumination is even more challenging when one is upset, angry, or captured by stressful life circumstances.

During conversations with students about PA, MM, and HRV-BF, there were reports that other approaches-such as singing, chanting, or toning were able to capture rapidly their attention and quiet the "busy-mind" more quickly. There has been some support for the hypothesis that vocalizations have a calming or soothing effect on strong negative emotional experiences. For example, preliminary research related to vagal nerve stimulation in patients with depression suggested that audible chanting of $O M$ vs. ssss (e.g., repeating the sound produced by uttering $O M$ or ssss, respectively) resulted in "significant deactivation observed bilaterally during 'OM' chanting in comparison to the resting brain state in orbito-frontal, anterior cingulate, parahippocampal gyri thalami and hippocampi" (Kalyani et al., 2011, p. 4). The authors speculated the sound vibrations stimulated the auricular branches of the vagal nerve with resultant effects on brain activation. Another study examining OM meditation using EEG spectral analysis found increases in theta waves across all brain regions, an indicator of enhanced relaxation (Harne \& Hiwale, 2018). Porges (2017) suggests that “...prosodic vocalizations (e.g., chants) in the frequency band that would overlap with the vocal signals of safety..." (p. 10) and "...by expanding the duration of exhalation and reducing the duration of inspiration..." (Porges, 2017, p. 16) have a positive effect on reducing emotional reactivity.

Whereas other studies have directed participants to rhythmically chant the sounds of $O M$ or repeatedly form the sound of ssss, this activity explored 1) the self-report of mindfulness and toning practice $U O$ (pronounced as you) as well as 2) the psychophysiology effects of mindfulness and toning practice. Toning as a practice can be distinguished from chanting or singing, where, for example, Snow, Bernardi, Sabet-Kassouf, Moran, and Lehman (2018) write: "Toning is a form of vocalizing that utilizes the natural voice to express sounds ranging from cries, grunts, and groans to open vowel sounds and humming on the full exhalation of the breath. Music therapists are increasingly utilizing toning in 
their clinical practice for a variety of therapeutic aims" (p. 221).

\section{Method}

\section{Observation 1}

Self-report of mindfulness and toning practice.

Subjects: 91 undergraduate college students (35 males, 51 females, and 5 unspecified; average age, 22.4 years, $S D=3.5$ years). As a report about an effort to improve the quality of a classroom activity, this report of findings was exempted from Institutional Review Board oversight.

Procedure: In the classroom, students sat comfortably in their chairs. Then the mindfulness and toning practices were explained, and the students were given an opportunity to ask questions. While sitting in class, students began either a 3-min mindfulness practice or a 3-min audible toning practice (vocalizing the sound of UO). They then filled out a subjective assessment form rating experiences of mind wandering, occurrence of intrusive thoughts, and sensations of vibration on a scale from 0 (not at all) to 10 (all the time) that occurred during the practice. They also rated experiences of peacefulness, relaxation, stress, warmth, anxiety, and depression from before to after the practice, as well as a description of prior experiences with depression and anxiety symptoms. The brief 3-min practices were counterbalanced with either mindfulness practice or audible toning, respectively, followed by completion of subjective rating forms.

\section{Observation 2}

Psychophysiology of mindfulness practice and toning practice.

Subjects: 11 undergraduate students (4 males, 7 females; average age 21.4 years).

Equipment: Physiological signals were recorded with an 8-channel polygraph (ProComp Infiniti system running Biograph Infiniti software version 6.1, Thought Technology, Ltd., Montreal, Canada). Respiration was monitored from the abdomen and upper thorax with strain gauges. Heart rate $(\mathrm{HR})$ was monitored with a blood volume pulse sensor placed on the thumb.

Procedure: After the sensors were attached, the subjects faced away from the screen, so they did not receive feedback. They then followed the same procedure as described above, with $3 \mathrm{~min}$ of mindfulness, or toning practice, counterbalanced. After each condition, they completed a subjective assessment form rating experiences as described above.

\section{Physiological Data Analysis}

The respiration signal was analyzed for breathing rate per minute. The blood volume pulse (BVP) signal, from which the HR was derived as the index of $\mathrm{HRV}$, is used to compute the standard deviation of $\mathrm{HR}$ in beats per minute and then expressed as the standard deviation of the normalized beat-tobeat interval (SDNN) measured in milliseconds (ms).

\section{Results}

\section{Subjective Results of Observation 1}

The participants reported less mind wandering during the toning condition $(M=3.7)$ than during meditation condition $(M=5.6)$ as determined by a single factor ANOVA, $F(1,179)=29.17, p<.001$; less intrusive thoughts during toning $(M=3.2)$ than during meditation $(M=4.7)$ as determined by a single factor ANOVA, $F(1,178)=14.56, p<.001$; and more awareness of body vibration during toning $(M=6.2)$ compared to the meditation condition ( $M=$ 2.3) as determined by a single factor ANOVA, $F(1$, $178)=104.03, p<.001$, as shown in Figure 1 .

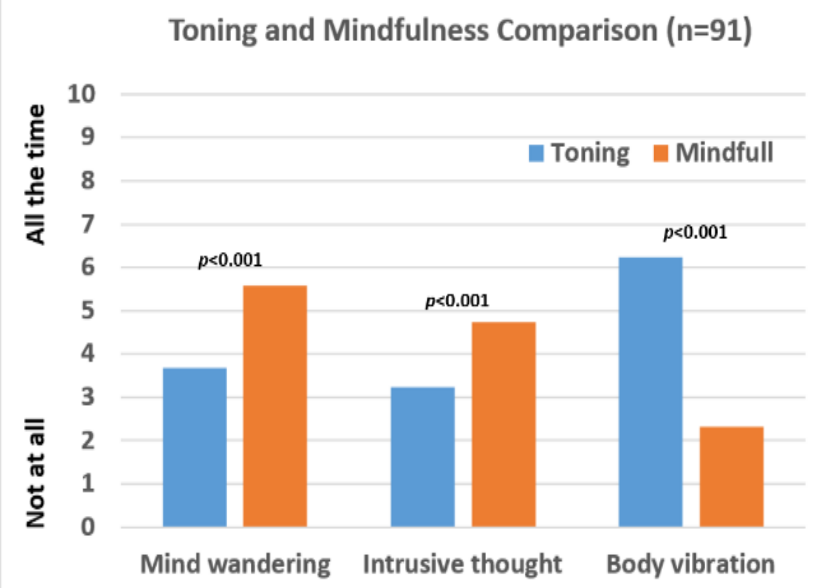

Figure 1. Differences between mindfulness and toning practice.

There was no statistically significant difference between the two practices in the increased selfreport of peacefulness, warmth, relaxation, and decreased self-report of anxiety and depression as shown in Figure 2. 


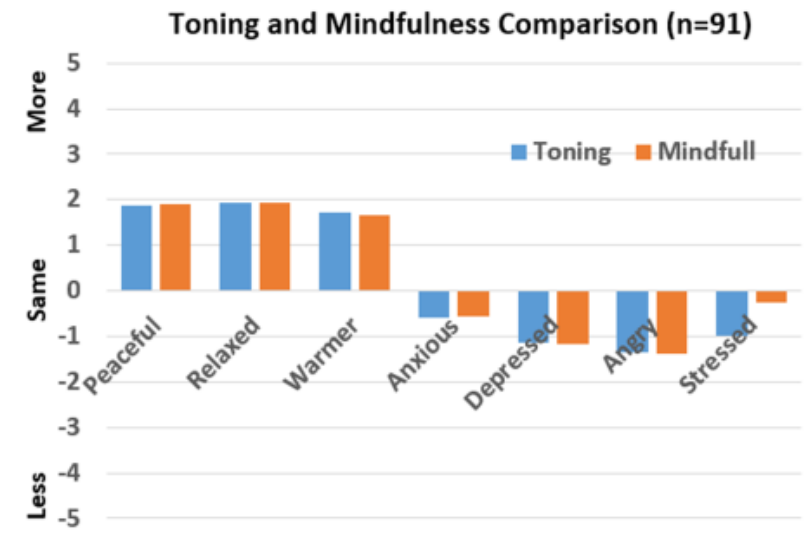

Figure 2. No significant difference between toning and mindfulness practice in relaxation or stress reports.

For the $30 \%$ of participants with the highest selfreported rating of depression, toning was more beneficial than mindfulness practice in reducing mind wandering as determined by a single factor ANOVA, $F(1,58)=6.30, p=.015$; as well as reducing intrusive thoughts as determined by a single factor ANOVA, $F(1,58)=6.20, p=.016$, as shown in Figures 3 and 4.

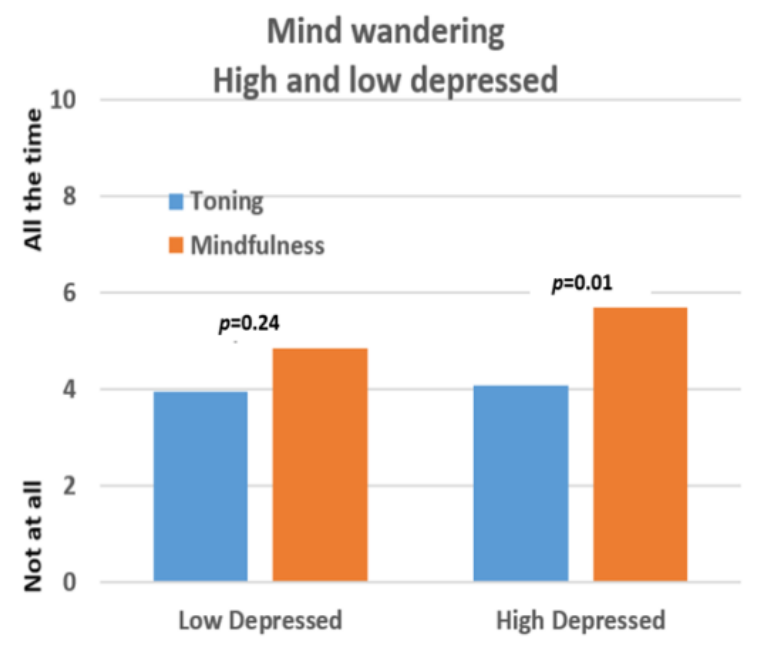

Figure 3. Effect of mindfulness and toning practice for high versus low reports of depression.

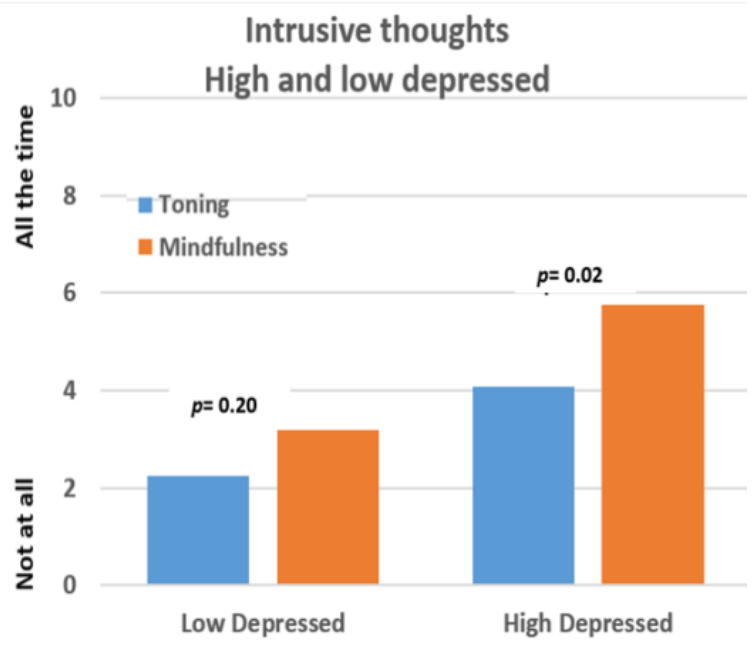

Figure 4. Effect of mindfulness and toning practice for high versus low reports of depression.

Physiological Results of Observation 2

There was a decrease in respiration rate during toning practice (TP, $4.6 \mathrm{br} / \mathrm{min}$ ) as compared to MP (11.6 br/min) as determined by a single factor ANOVA, $F(1,18)=30.84, p<.001)$; as well as an increase in HR standard deviation (SDNN) during the toning condition (11.6; SDNN $103.7 \mathrm{~ms}$ ) as compared to the mindfulness condition (6.4; SDNN $61.9 \mathrm{~ms}$ ) as determined by a single factor ANOVA, $F(1,20)=11.86, p=.002)$. Two representative, counter-balanced recordings are shown in Figure 5. For both sample recordings, during toning the respiration rate (abdomen and chest) decreases. Furthermore, in these samples the HR increases much more during inhalation and decreases much more during exhalation, as compared to mindfulness portions of the recordings, and with the pre and post baselines. 

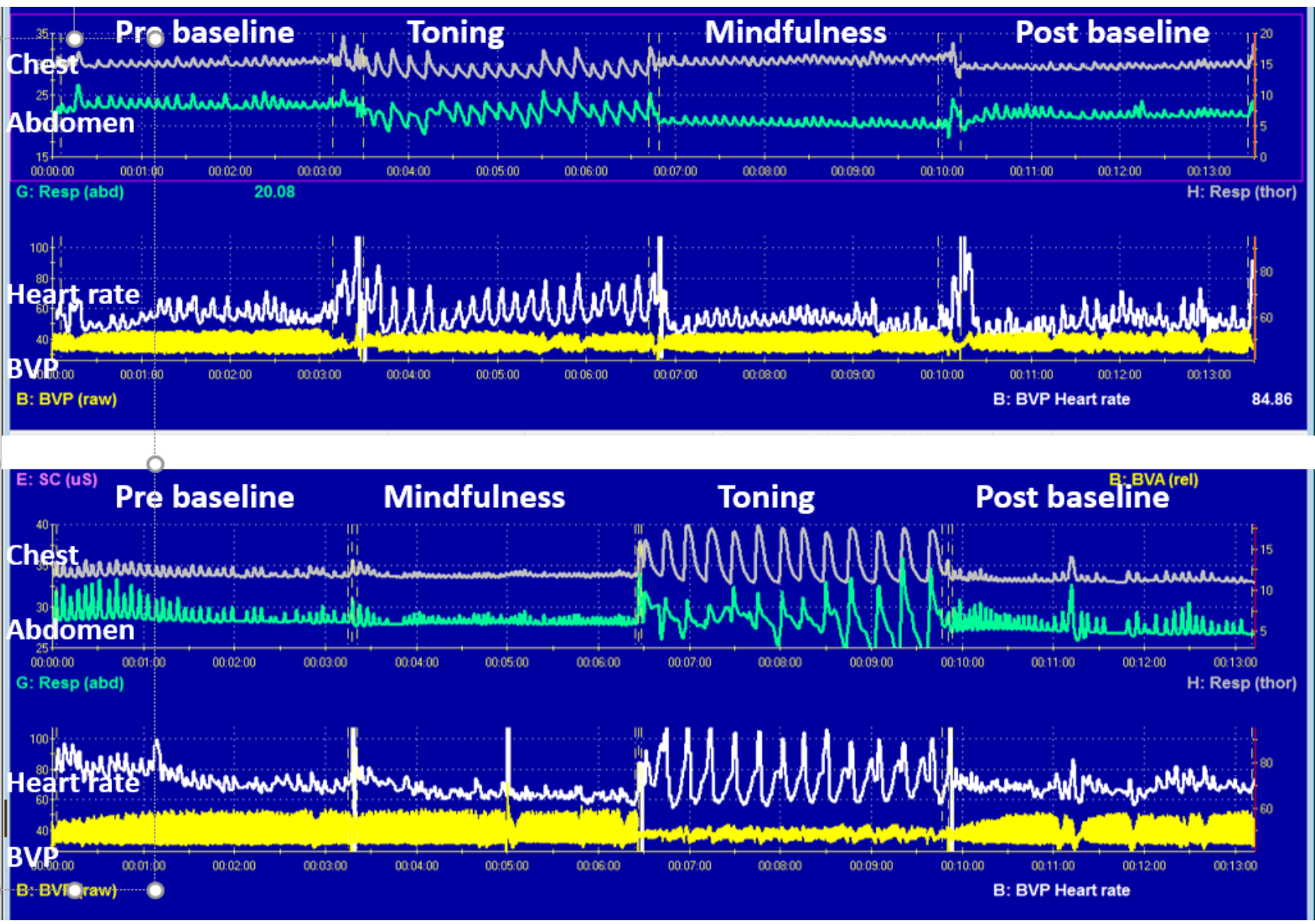

Figure 5. Two participants' representative recordings of breathing and heart rate during mindfulness and toning practice.

\section{Discussion}

The observations suggest that a brief 3-min TP is a useful strategy to reduce mind wandering as well as inhibit intrusive thoughts and increase HRV. We recommend that when patients report feeling worried and anxious that they first practice toning before beginning bio-neurofeedback training.

TP very rapidly reduced respiration rate and increased HRV. The increase in HRV and slow respiration during TP occurred along with selfreported reductions in mind wandering and intrusive thoughts, as well as increases in awareness of somatic sensations such as body vibrations. It will be a useful strategy to practice toning as a complement to other bio-neurofeedback protocols, including biofeedback-assisted HRV training, especially because focusing on the toning appears to facilitate HRV increases without striving.
It is recommended that when clients report excessive mind wandering and intrusive thoughts, that they practice toning as the first intervention before beginning the MP, relaxation or bioneurofeedback training. By combining toning as a rapid thought-reducing strategy and mindfulnessbased stress reduction as a long-term skill acquisition practice, clinical outcomes may improve. Toning is a portable skill that clients can implement immediately to inhibit intrusive thoughts and to enhance parasympathetic tone.

There are some limitations of these observations. The data represents a student sample that may not generalize to other populations. The audible toning also took place in a group setting; thus, effects may vary if done alone or with a facilitator. There are enough encouraging observations to suggest that more research is needed to explore the value of TPs as a brief intervention for practitioners to use during biofeedback/neurofeedback sessions. 
Future research directions include exploring conditions that are similar to ruminative thoughts. For example, as described by Smith and Alloy (2009), other disruptive thought processes may be at work with labels such as negative automatic thoughts, private self-consciousness, self-focused attention, repetitive thoughts, intrusive thoughts, obsessions, worry, emotion regulation and coping, and neuroticism. Furthermore, future research may explore conditions that distinguish frequency, intensity, and duration of toning, chanting, singing, and other vocal productions that affect affective states. Regardless of what future discoveries await, TPs appear to be a simple and easy strategy for reducing intrusive thoughts and improving cardiorespiratory balance.

\section{Author Disclosure}

Authors have no grants, financial interests, or conflicts to disclose.

\section{References}

Conder, R. L., \& Conder, A. A. (2014). Heart rate variability interventions for concussion and rehabilitation. Frontiers in Psychology, 5, 890. https://doi.org/10.3389/fpsyg.2014.00890

Creswell, J. D. (2015). Mindfulness Interventions. Annual Review of Psychology, 68, 491-516. https://doi.org/10.1146/annurevpsych-042716-051139

Dahl, C. J., Lutz, A., \& Davidson, R. J. (2015). Reconstructing and deconstructing the self: Cognitive mechanisms in meditation practice. Trends in Cognitive Sciences, 19(9), 515-523. https://doi.org/10.1016/j.tics.2015.07.001

Ginsberg, J. P., Berry, M. E., \& Powell, D. A. (2010). Cardiac coherence and posttraumatic stress disorder in combat veterans. Alternative Therapies, 16(4), 52-60. https://pdfs.semanticscholar.org/6d27/e5a6084c5d824eca7e5 0c009413a912f7bf9.pdf?_ga=2.186430496.887517633.1561 505930-1100946524.1561505930

Goessl, V. C., Curtiss, J. E., \& Hofmann, S. G. (2017). The effect of heart rate variability biofeedback training on stress and anxiety: A meta-analysis. Psychological Medicine, 47(15), 2578-2586. https://doi.org/10.1017 /S0033291717001003

Harne, B. P., \& Hiwale, A. S. (2018). EEG spectral analysis on OM mantra meditation: A pilot study. Applied Psychophysiology and Biofeedback, 43(2), 123-129. https://doi.org/10.1007/s10484-018-9391-7

Hofmann, S. G., \& Gómez, A. F. (2017). Mindfulness-based interventions for anxiety and depression. Psychiatric Clinics of North America, 40(4), 739-749. https://doi.org/10.1016 /j.psc.2017.08.008

Kalyani, B. G., Venkatasubramanian, G., Arasappa, R., Rao, N. P., Kalmady, S. V., Behere, R. V., ... Gangadhar, B. N. (2011). Neurohemodynamic correlates of 'OM' chanting: A pilot functional magnetic resonance imaging study. International Journal of Yoga, 4(1), 3-6. https://doi.org/10.4103/0973-6131.78171
Khazan, I. Z. (2013). The clinical handbook of biofeedback: $A$ step-by-step guide for training and practice with mindfulness. Chichester, West Sussex, UK: John Wiley \& Sons.

Khazan, I. Z. (2019). Biofeedback and Mindfulness in Everyday Life. New York, NY: W. W. Norton \& Company.

Kopelman-Rubin, D., Omer, H., \& Dar, R. (2017). Brief therapy for excessive worry: Treatment model, feasibility, and acceptability of a new treatment. Journal of Psychotherapy Integration. http://dx.doi.org/10.1037/int0000100

Lagos, L., Thompson, J., \& Vaschillo, E. (2013). A preliminary study: Heart rate variability biofeedback for treatment of postconcussion syndrome. Biofeedback, 41(3), 136-143. https://doi.org/10.5298/1081-5937-41.3.02

Lehrer, P. M., \& Gevirtz, R. (2014). Heart rate variability biofeedback: How and why does it work? Frontiers in Psychology, 5, 756. https://doi.org/10.3389/fpsyg.2014.00756

McGrady, A., \& Moss, D. (2018). A pathways approach to chronic pain. In A. McGrady \& D. Moss, Integrative Pathways (pp. 111-129). Cham, Switzerland: Springer International Publishing. https://doi.org/10.1007/978-3-319-89313-6_6

Ost, L. G. (2008). Efficacy of the third wave of behavioral therapies: A systematic review and meta-analysis. Behaviour Research and Therapy, 46(3), 296-321. https://doi.org /10.1016/j.brat.2007.12.005

Porges, S. W. (2017). Vagal pathways: Portals to compassion. The Oxford Handbook of Compassion Science, 189-204.

ProComp Infiniti 8-channel system with Biograph Infiniti software (Version 6.1) [Apparatus]. Montreal, Canada: Thought Technology, Ltd.

Renna, M. E., Quintero, J. M., Fresco, D. M., \& Mennin, D. S. (2017). Emotion regulation therapy: A mechanism-targeted treatment for disorders of distress. Frontiers in Psychology, 8, 98. https://doi.org/10.3389/fpsyg.2017.00098

Shaffer, F., \& Ginsberg, J. P. (2017). An overview of heart rate variability metrics and norms. Frontiers in Public Health, 5, 258. https://doi.org/10.3389/fpubh.2017.00258

Smith, J. M., \& Alloy, L. B. (2009). A roadmap to rumination: A review of the definition, assessment, and conceptualization of this multifaceted construct. Clinical Psychology Review, 29(2), 116-128. https://doi.org/10.1016 /j.cpr.2008.10.003

Snow, S., Bernardi, N. F., Sabet-Kassouf, N., Moran, D. \& Lehmann, A. (2018). Exploring the Experience and Effects of Vocal Toning. Journal of Music Therapy, 55(2), 221-250. https://doi.org/10.1093/jmt/thy003

Thompson, M., \& Thompson, L. (2015). The Neurofeedback Book: An Introduction to Basic Concepts in Applied Psychophysiology. Wheat Ridge, CO: Association for Applied Psychophysiology and Biofeedback.

van der Zwan, J. E., de Vente, W., Huizink, A. C., Bogels, S. M., \& de Bruin, E. I. (2015). Physical activity, mindfulness meditation, or heart rate variability biofeedback for stress reduction: A randomized controlled trial. Applied Psychophysiology and Biofeedback, 40(4), 257-268. https://doi.org/10.1007/s10484-015-9293-x

Received: May 22, 2019

Accepted: July 31,2019

Published: September 13, 2019 